\title{
ProSPer: a MOST model extension applied to persuasive interactive systems
}

\author{
Alessandro Fenicio, Gaëlle Calvary, Yann Laurillau \\ Univ. Grenoble Alpes, LIG, F-38000 Grenoble, France \\ CNRS, LIG, F-38000 Grenoble, France \\ alessandrofenicio@gmail.com, \\ Gaelle.Calvary@imag.fr, \\ Yann.Laurillau@imag.fr
}

\author{
Jean Vanderdonckt \\ Université catholique de Louvain, \\ Louvain School of Management \\ Place des Doyens, 1 - 1348 Louvain-la-Neuve \\ jean.vanderdonckt@uclouvain.be
}

\begin{abstract}
Developing persuasive interactive system faces the challenge of describing multiple-domain goals to be achieved, along with an context-aware driving of persuasion. We developed ProSPer, a model capable of dealing with this challenges and we implemented two experiments using an Android application and a selfmonitoring bracelet. The preliminary results shows that participants understood and applied effectively the model, giving further design prospectives of research and development.
\end{abstract}

Context-Aware persuasive interactive systems, MOST model, ProSPer

\section{INTRODUCTION}

"Smoking kills", "Drive or Drink" or Avoid eating junk food. Advertisements become more and more direct, unfortunately do not deal with an effective change in the behaviour and habits. This change involves a more complex process (a first commitment, a durable engagement and long-term maintenance) theorized and implemented by the field of Persuasive Technology [IJsselsteijn et al. (2016)].

In this work, we deal with two research questions: the first is about "how to provide users the capability of describing the multiple-domain changes they want to achieve", and second "how driving them a contextaware effective persuasion".

In order to deal with multiple-domain changes (e.g. health, productivity, security...), we promote a new approach based on the MOST model inspired from business analysis, whose models are expected to cover a wide range of heterogeneous application domains (e.g., employees management motivation or productivity, general management, marketing strategies).

On the other hand, in order to drive a context-aware persuasion, we propose the ProSPer model as an extension of MOST model, reorganized in layers (problem, solution, persuasion) permitting a contextaware operationalisation of the design principles in the field of persuasion. The context implemented by ProSPer is intended as "any information that can be used to characterize the situation of an entity" [Dey, Anind K. (2001)], and is characterized by the available sensors present on the platform of use.

\section{EXTENDING MOST WITH PROSPER}

\subsection{Most applied to behavioral change}

MOST analysis is a top-down technique used to analyse what and how has to be achieved. It stands for Mission (the rationale and direction for the organisation), Objective (the goals that the organisation aims to achieve), Strategy (the mediumto long-term plans and actions that will enable the organisation to achieve its objectives) and Tactics (the detailed, short-term plans and actions that will deliver the strategy) [ Cadle et al. (2014)][ Sondhi, Rakesh (1999)]

We promote an adaptation of the statement of Cadle et al (2014). Thus, we define the Mission (e.g. Be in shape) as the final change a person wants to achieve completing a set of Objectives (e.g. eating healthy, Doing sports). The strategies are the solutions that the person chooses to apply according to his/her own profile (e.g., eating an apple, swimming) while the Tactics refers to the context in which the person decides to apply a given Strategy (e.g. twice a week).

Along with the Mission "Be in Shape" (related to health) we can have other mission as for example "Improve my time Management" (related 


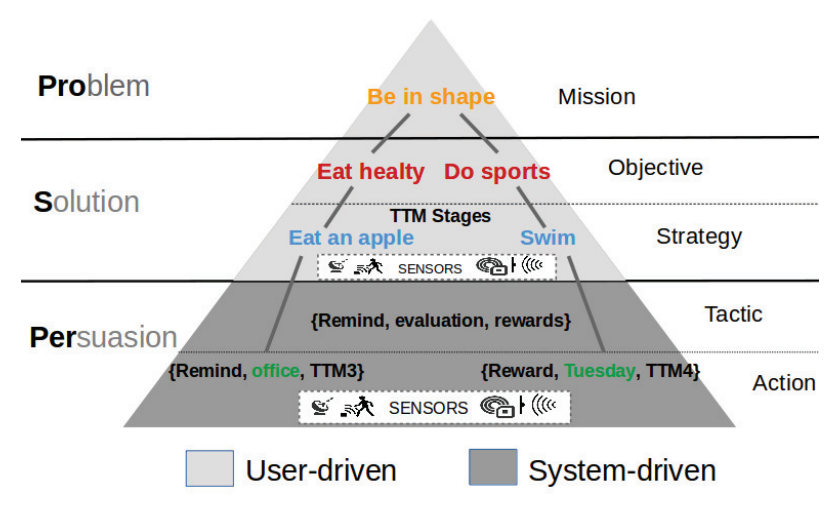

Figure 1: ProSPer Model applied on a user scenario

to productivity) with other relative Objectives, Strategies and Tactics.

\subsection{ProSPer}

Figure 1, gives an overview of the ProsPer layers applied on a user scenario. ProSPer is divided into three layers describing the persuasion applied (persuasion layer) to the solutions (solution layer) found by the user for a given problem (problem layer). The first two layers are related to the structuring of the Problem and Solution in the MOST paradigm while the last is responsible for driving the persuasion to the end user. In our vision, the extra part added to the MOST model is first given by the sensors, able to detect the context of use, and then by the concept of Actions describing applicability of persuasion techniques to a given context of use.

Instantiating ProSPer on the example "Be in shape" consists in regrouping the Mission in the Problem layer and the Objectives along with Strategies in the Solution layer, as pictured in Figure 1. The Objective is refined with the stage of the change in which the user is found, such as the ones defined by the transtheoretical model of behaviour change[Prochaska and Velicer (1997)]. In this prospective the Objective "Eating healthy" is enriched by the one of the status "Contemplation, Preparation, Action or Maintenance" according to how the user is living the change. In the persuasive layer along with the Tactics we have the Actions, used to describe in which form the persuasion will be presented to the user (remind, suggest and reward)[Oinas-Kukkonen ans Harjumaa (2008)]. Actions take place according to two factors: the stage of change of the user, and the context given by sensors, which can be of diverse type (Space, Time, RFID) dependently on the platform in use.

We currently have designed and developed a multiple-domain software environment according to ProSPer. Such environment has been used to conduct user studies further detailed.

\section{USER STUDIES}

We conduct a study to determine how MOST applied to persuasion is relevant. The survey involved 12 participants of age between 22-53 of mixed occupation. They were split into two groups taking part to different experiments lasting for five days and further evaluated using a questionnaire, protocol already used to evaluate Behaviour Change Support Systems by Piiastiina et al. (2016).

The first group had to wear a string bracelet with color stripes describing the engaged Missions with Objectives, Strategies and Tactics. The bracelet implemented the implicit action "Reminding" to users the engagement using a self-monitoring approach.

The second group used an Android application, based on our ProSPer-based multiple-domain software. This application permits to execute the same task in a digital implementation based on a context aware persuasion taking advantage of the sensors available on the smart-phone (GPS, time). The implementation was based on the transtheoretical model of behaviour change: several levels of engagement (corresponding to the stages of the model) allowed the user passing among different configurations of the three persuasive tactics we used (remind, suggest and reward).

Finally the IBM CSUQ debriefing questionnaire by [Lewis and James (1995)] was given to participant to understand their capability in applying the model to the behaviour change they targeted (e.g. MOST applied to persuasion).

\section{RESULTS AND PERSPECTIVES}

The reported results represent the average consensus on a given affirmation $\bar{x}$ and its standard deviation $\sigma$ on a scale of 7 . The trends indicates that participants said to be "able to apply the model" on their examples $(\bar{x}=6,2 \pm 0,63, \sigma=1,03)$ and to be "overall satisfied" by the experiment that "triggered them to act", "remembering the model" $(\bar{x}=5,40 \pm$ $0,72, \sigma=1,17)$. Using the Android application, participants found that the system was "effectively helping" them in the change $(\bar{x}=5,40 \pm 0,71, \sigma=$ 0,81 ), evidencing that the context-aware persuasion was more effective than a simple self-monitoring strategy. Participants gave also additional requirements for the application as a more diverse set of persuasive message to be notified. The overall trend indicates the interest of participants in having an allin-one installed on their phones application instead of several ones to reach the behavioural change they target. This study seems to confirm our approach based on MOST. We consider much more long-term studies in a near future. 


\section{REFERENCES}

IJsselstei W., de Kort, Y., Midden, C., Eggen, B., andvan den Hoven, E. Persuasive Technology: International Conference on Persuasive Technology for Human Well-Being PERSUASIVE 2006 Proceedings,vol. 3962. Springer, 2006.

James Cadle, Debra Paul, Paul Turner (2014) Business Analysis Techniques: 99 Essential Tools for Success (2th edn). BCS, The Chartered Institute for IT.

Oinas-Kukkonen, H., and Harjumaa, M. A systematicframework for designing and evaluating persuasive systems. In Persuasive technology. Springer, (2008)

Sondhi and Rakesh Total Strategy. Airworthy (1999)
Piiastiina Tikka, Bereket Woldemicael, and Harri Oinas-KukkonenBuilding an App for Behavior Change: Case RightOnTime Fourth International Workshop on Behavior Change Support Systems (BCSS 16)

Prochaska, J. O., and Velicer, W. F. The transtheoretical model of health behavior change. American journal of health promotion(1997).

Dey, Anind K. Understanding and using context. Personal and ubiquitous computing 5.1 (2001).

Lewis, James R. IBM computer usability satisfaction questionnaires: psychometric evaluation and instructions for use.. International Journal of Human Computer Interaction 7.1 (1995). 